\section{THU0560 DISTINCTIVE IMAGING FEATURES BETWEEN IGG4- RELATED OPHTHALMIC DISEASE AND GRAVES' ORBITOPATHY: A COMPARATIVE STUDY}

Eduardo Martin-Nares ${ }^{1}$, Oziel Amaya-Piña ${ }^{2}$, Rosa Delia Delgado-Hernández ${ }^{2}$, Gabriela Hernandez-Molina ${ }^{1} .{ }^{1}$ Instituto Nacional de Ciencias Médicas y Nutrición Salvador Zubirán, Immunology and Rheumatology, Mexico City, Mexico; ${ }^{2}$ Instituto Nacional de Ciencias Médicas y Nutrición Salvador Zubirán, Department of Radiology and Molecular Medicine, Mexico City, Mexico

Background: IgG4-related ophthalmic disease (IgG4-ROD) may present as a cause of orbital myositis leading to proptosis and diplopia. This clinical scenario can be mistakenly diagnosed as Graves' orbitopathy (GO), preventing a timely and adequately treatment.

Objectives: To elucidate if there are specific radiological features that might differentiate between IgG4-ROD and GO by imaging.

Methods: We included 19 patients with diagnosis of lgG4-related disease (IgG4-RD) according to the Comprehensive Diagnostic Criteria for IgG4$\mathrm{RD}$, who regularly attended a tertiary referral center in Mexico City. All the patients had ophthalmic involvement and available computed tomography (CT) and/or magnetic resonance imaging (MRI) of the orbits. We also included 32 patients with GO with available CT and/or MRI of the orbits. Imaging studies were evaluated by a blinded head and neck radiologist for the following features: exophthalmos, extraocular muscles (EOM) size and morphology, lacrimal gland enlargement, orbital fat involvement, stretching of the optic nerve (ON), ON sheath thickening and orbital bone changes.

Results: Both groups were similar in age $(49.1 \pm 15.8$ vs. $51.6 \pm 14.7$, $\mathrm{p}=0.58$ ) and gender (men $58.9 \%$ vs. $40.6 \%, \mathrm{p}=0.23$ ). In addition to ophthalmic involvement, $18(94.7 \%)$ IgG4-RD patients had extra-ophthalmic involvement with a median number of organs involved of 7 (1-12), mainly submandibular glands $(73.7 \%)$, lymph nodes $(68.4 \%)$, parotid glands $(63.2 \%)$ and pancreas (47.4\%). Three patients were misdiagnosed as GO before IgG4-RD diagnosis. Graves' disease was the underlying thyroid disorder in $28(87.5 \%)$ GO patients, Hashimoto's thyroiditis in $2(6.3 \%)$, papillary thyroid carcinoma in one $(3.1 \%)$ and one patient was euthyroid with positive thyroid stimulating immunoglobulin. The prevalence of exophaltmos $(78.9 \%$ vs. $93.8 \%)$, bilateral involvement $(78.9 \%$ vs. $87.8 \%)$ and overall EOM involvement (47.4\% vs. $68.8 \%$ ) was similar between IgG4ROD and GO groups. However, IgG4-ROD patients had a higher frequency of lacrimal gland involvement $(73.7 \%$ vs. $10.7 \%, p=0.001)$ and a tendency for the lateral rectus to be the most frequently involved EOM $(22.2 \%$ vs. $0 \%, p=0.07)$; conversely they had a lower prevalence for the inferior rectus to be the most frequently involved EOM, $(33.3 \%$ vs. $72.7 \%, p=0.04)$, orbital fat involvement $(47.4 \%$ vs. $81.3 \%, p=0.01)$, ON stretching $(57.9 \%$ vs. $87.5 \%, p=0.02)$ and orbital bone changes $(0 \%$ vs. $25 \%, p=0.02$ ). EOM bellies were involved in all the IgG4-ROD and GO cases, whereas EOM tendon involvement was present in $9 \%$ of $\mathrm{GO}$ and in none of the IgG4-ROD group. Patients with IgG4-ROD had more frequently the combination of lacrimal gland and lateral rectus $(31.6 \%$ vs. $3.1 \%, p=0.008$ ) and less frequently the combination EOM and orbital fat involvement $(21.2 \%$ vs. $59.4 \%, p=0.008)$.

At the logistic regression analysis we found an association of lacrimal gland involvement (OR 64.4.0, 95\% Cl 6.8-609.5, $\mathrm{p}=0.001)$ with IgG4ROD. In a second model including combined variables, the combination of lacrimal gland and lateral rectus involvement was associated with IgG4-ROD (OR 62.5, 95\% Cl 3.31-1000), P=0.006), whereas the presence of EOM and orbital fat involvement was protective (OR 0.05, 95\% Cl 0.006-0.48, $\mathrm{p}=0.009$ ).

Conclusion: Imaging features may reliably differentiate between IgG4-ROD and GO. The presence of both lacrimal gland and lateral rectus enlargement must alert clinicians to consider IgG4-ROD diagnosis.

\section{REFERENCES:}

[1] Tiegs-Heiden CA, Eckel LJ, Hunt CH, et al. Immunoglobulin G4-related disease of the orbit: imaging features in 27 patients. AJNR Am J Neuroradiol. 2014 Jul;35(7):1393-7.

Disclosure of Interests: None declared

DOI: 10.1136/annrheumdis-2019-eular.5144

\section{THU0561 \\ CANAKINUMAB FOR THE TREATMENT OF ADULT ONSET STILL'S DISEASE TO ACHIEVE REDUCTION OF ARTHRITIC MANIFESTATION AT WEEK 12: AN INVESTIGATOR-INITIATED MULTI-CENTRE, PLACEBO- CONTROLLED STUDY (CONSIDER)}

Claudia Kedor ${ }^{1}$, Joachim Listing ${ }^{2}$, Jan Zernicke ${ }^{1}$, Anja Weiß ${ }^{2}$, Frank Behrens ${ }^{3}$, Norbert Blank ${ }^{4}$, Jörg Henes ${ }^{5}$, Jörn Kekow ${ }^{6}$, Andrea Rubbert-Roth ${ }^{7}$, Hendrik Schulze-Koops ${ }^{8}$, Eva Seipelt ${ }^{9}$, Christof Specker ${ }^{10}$, Eugen Feist ${ }^{1}$, The Study was supported by Novartis Germany. ${ }^{1}$ Charité - Universitätsmedizin Berlin, Rheumatology and clinical Immunology, Berlin, Germany, ${ }^{2}$ Deutsches RheumaForschungszentrum (DRFZ), Epidemiology, Berlin, Germany; ${ }^{3}$ Goethe University Frankfurt, Fraunhofer Institut für Molekularbiologie und Angewandte Onkologie IME, Frankfurt a.M., Germany; ${ }^{4}$ University Hospital Heidelberg, Internal Medicine 5, Rheumatology, Heidelberg, Germany; ${ }^{5}$ University Hospital Tuebingen, Centre for Interdisciplinary Clinical Immunology, Rheumatology and Auto-inflammatory Diseases (INDIRA) and Internal Medicine II, Tübingen, Germany; ${ }^{6} \mathrm{Helios}$ Fachklinik Vogelsang-Gommern, Klinik für Rheumatologie, 39245 VogelsangGommern, Germany; ${ }^{7}$ Kantonsspital St Gallen, Klinik für Rheumatologie, St Gallen, Switzerland; ${ }^{8}$ University of Munich, Department of Internal Medicine IV, Divison of Rheumatology, München, Germany, ${ }^{9}$ Immanuel Krankenhaus Berlin, Rheumatologie und Klinische Immunologie, Berlin, Germany, ${ }^{10}$ Evangelisches Krankenhaus • Kliniken Essen-Mitte, Klinik für Rheumatologie and Klinische Immunologie, Essen, Germany

Background: Inhibition of interleukin-1 (IL-1) represents a promising treatment option in adult-onset Still's disease (AOSD). Canakinumab (CAN) is approved for treatment of systemic juvenile idiopathic arthritis (sJIA) since 2013 and recently for AOSD in Europe. In SJIA, CAN has a proven marked impact on systemic and articular disease activity, but for AOSD RCT data is limited.

Objectives: Investigation of efficacy and safety of CAN in AOSD patients with joint involvement by means of a multi-centre, double-blinded, randomized, placebo controlled trial.

Methods: AOSD patients with joint involvement (tender and swollen joint count $\geq 4$ each) were stratified by pre-treatment status with biologics and randomized in a ratio of $1: 1$ to CAN (4 mg/kg up to a maximum of 300 $\mathrm{mg}$ ) or placebo (PBO) s.c. every 4wks. The primary endpoint was defined as the proportion of patients with a clinical-relevant reduction $(\geq 1.2)$ in disease activity as measured by change in disease activity score (DAS28) at week 12. There were two major protocol violations: two PBO patients received blinded CAN at week 4. Therefore, a per-protocol (PP) analysis, which excluded data of these two patients, was performed.

Results: The study was prematurely stopped with 35 out of 68 planned patients enrolled due to approval of CAN for AOSD by EMA. At enrollment, mean DAS28 represented high disease activity for both: 5.4 in CAN $(n=18)$ and 5.3 in PBO $(n=17)$. In the intention-to-treat analysis, 12 $(67 \%)$ CAN and $7(41 \%)$ PBO patients met the primary endpoint. The $25.5 \%[95 \% \mathrm{Cl}:-10.3 \% ; 55.9 \%]$ difference in the response rate did not achieve statistical significance $(\mathrm{p}=0.18)$. Using the PP analysis, the treatment difference for primary endpoint was $33.4 \%[95 \% \mathrm{Cl}$ : $-10.3 \% ; 55.9 \%$, $p=0.08]$. We observed higher ACR50 (50\% CAN vs. 6.7\% PBO, $p=$ 0.009 ), ACR70 (28\% CAN vs. 0\% PBO, $p=0.049$ ) response rates, (PP analysis). Higher mean improvements in the CAN vs PBO group for DAS28(ESR and CRP), ESR, CRP, 66 swollen and 68 tender joint counts at week 12 were observed (PP analysis). There were 47 and 21 non-serious adverse events (AE) reported in CAN and PBO groups, respectively. Two CAN patients experienced an SAE (hepatotoxicity and patellofemoral pain syndrome).

Conclusion: Although the study was terminated prematurely and the primary endpoint did not achieve statistical significance, treatment of patients with active AOSD with CAN led to improvement of several outcome measures. In addition, safety profile was similar to that reported in SJIA and no unexpected safety issues were observed. This data support the treatment of AOSD patients with CAN.

\section{REFERENCES:}

[1] Ruperto. NEJM (2012): 2396-2406

Disclosure of Interests: Claudia Kedor Grant/research support from: Sobi, Consultant for: Novartis, Speakers bureau: Novartis, Roche, Joachim Listing: None declared, Jan Zernicke: None declared, Anja Weiß: None declared, Frank Behrens Grant/research support from: AbbVie, Pfizer Roche, Chugai, Prophylix, Bioline, Novartis, Consultant for: AbbVie, Pfizer, Roche, Chugai, UCB, Bristol-Myers Squibb, Celgene, MSD, Novartis, Biotest, Janssen, Genzyme, Eli Lilly, Speakers bureau: Ad board: AbbVie, Pfizer, Roche, Chugai, UCB, Bristol-Myers Squibb, Celgene, Novartis, Biotest, Janssen, Genzyme, Eli Lilly, Norbert Blank Grant/research support 